\title{
Comparação dos resultados da fala após as cirurgias de retalho faríngeo e veloplastia intravelar para correção da disfunção velofaríngea
}

\author{
Comparison of speech results following pharyngeal flap \\ and intravelar veloplasty for correction of velopharyngeal \\ dysfunction
}

\author{
Cíntia Yumi Mituuti ${ }^{1}$, Cristina Guedes de Azevedo Bento-Gonçalves ${ }^{2}$, \\ Silvia Helena Alvarez Piazentin-Penna ${ }^{3}$, Giovana Rinalde Brandã $0^{4}$, Cláudia Tiemi Mituuti ${ }^{5}$
}

\begin{abstract}
RESUMO
Objetivo: Comparar a fala e o funcionamento velofaríngeo após as técnicas de retalho faríngeo e veloplastia intravelar para a correção da disfunção velofaríngea residual. Métodos: Foi realizado um estudo retrospectivo com análise de 148 casos com fissura labiopalatina operada e submetidos à correção cirúrgica da disfunção velofaríngea, sendo 77 com retalho faríngeo (média de idade: 20,4 anos) e 71 com veloplastia intravelar (média de idade: 16,2 anos). Foram avaliadas a ressonância da fala, a presença de articulações compensatórias, a emissão de ar nasal e a extensão da falha no fechamento velofaríngeo antes e após as duas técnicas. Resultados: Dos 77 casos submetidos ao retalho faríngeo 64 (83\%) apresentaram melhora na ressonância, enquanto que dos 71 casos com veloplastia intravelar 48 (68\%) revelaram melhora, havendo diferença significativa entre os grupos. No grupo com retalho faríngeo, seis $(8 \%)$ apresentaram melhora na articulação compensatória e dois (3\%) no grupo veloplastia intravelar, enquanto a emissão de ar nasal melhorou em 17 (22\%) casos com retalho faríngeo e em 18 (26\%) com veloplastia intravelar. Não houve diferença entre os grupos quanto à articulação compensatória e emissão de ar nasal. A falha no fechamento velofaríngeo reduziu em 75 (96\%) casos com retalho faríngeo e 46 (66\%) com a veloplastia intravelar, havendo diferença entre os grupos. Conclusão: A técnica de retalho faríngeo mostrou-se mais efetiva na melhora da ressonância e no fechamento velofaríngeo quando comparada à veloplastia intravelar.
\end{abstract}

Descritores: Fenda labial/cirurgia; Fissura palatina/cirurgia; Insuficiência velofaríngea/terapia; Distúrbios da fala; Palato/cirurgia

\section{INTRODUÇÃO}

Um dos principais objetivos da equipe que trabalha com a

Trabalho realizado no Curso de Especialização em Motricidade Orofacial do Hospital de Reabilitação de Anomalias Craniofaciais, Universidade de São Paulo - USP - Bauru (SP), Brasil.

(1) Associação dos Pais e Amigos dos Exepcionais de Jundiaí - APAE Jundiaí (SP), Brasil.

(2) Setor de Fonoaudiologia do Hospital de Reabilitação de Anomalias Craniofaciais, Universidade de São Paulo - USP - Bauru (SP), Brasil.

(3) Diretoria Técnica dos Serviços Complementares do Hospital de Reabilitação de Anomalias Craniofaciais, Universidade de São Paulo - USP - Bauru (SP), Brasil.

(4) Setor de Fonoaudiologia do Hospital de Reabilitação de Anomalias Craniofaciais, Universidade de São Paulo - USP - Bauru (SP), Brasil.

(5) Programa de Pós-graduação (Mestrado) em Fonoaudiologia da Faculdade de Odontologia de Bauru, Universidade de São Paulo - USP - Bauru (SP), Brasil. Endereço para correspondência: Cristina Guedes de Azevedo BentoGonçalves. R. Silvio Marchione, 3-20, Vila Universitária, Bauru (SP), Brasil, CEP:17043-900. E-mail: mcguedes@usp.com.br

Recebido em: 25/08/2009; Aceito em: 13/7/2010 reabilitação de indivíduos com fissura labiopalatina é proporcionar-lhes uma fala normal. Para que isto ocorra, é necessário que o paciente seja submetido à palatoplastia primária antes da aquisição da fala, ou no período inicial de seu desenvolvimento. A correção cirúrgica da fissura palatina tem o objetivo de restabelecer a integridade anatômica e funcional do palato, a fim de possibilitar a separação das cavidades oral e nasal durante a fala. Entretanto, não basta apenas reparar a fenda palatina, sendo também necessário promover a função adequada do mecanismo velofaríngeo para a fala, resultando em pressão aérea intra-oral correta e ressonância equilibrada ${ }^{(1-3)}$.

O mecanismo velofaríngeo é formado pelo véu palatino e pelas paredes laterais e posterior da faringe que se movimentam conjuntamente, proporcionando o fechamento deste esfíncter durante a deglutição, o sopro e a emissão dos sons orais da fala. O padrão deste fechamento dependerá do grau de deslocamento de cada uma dessas estruturas durante a fala ${ }^{(4)}$. Quando há uma falha neste fechamento após a palatoplastia, com consequente passagem de ondas sonoras para a cavidade 
nasal, estamos diante de um quadro denominado de disfunção velofaríngea (DVF), que ocorre em aproximadamente $25 \%$ dos casos após a palatoplastia primária ${ }^{(5)}$. A causa mais frequente a DVF é a fissura palatina. Em muitos casos, mesmo após a correção cirúrgica primária do palato, os sintomas de DVF não são eliminados, sendo então necessária uma cirurgia secundária do palato ${ }^{(6,7)}$.

Os efeitos primários da DVF na fala são: a hipernasalidade, a emissão de ar nasal e o enfraquecimento dos fonemas de pressão, enquanto que os distúrbios articulatórios compensatórios são considerados efeitos secundários. A hipernasalidade caracteriza-se pelo acoplamento anormal das cavidades oral e nasal durante a produção das vogais, levando a uma alteração que é popularmente descrita como "voz fanhosa". Já a emissão de ar nasal é a passagem anormal de ar para a cavidade nasal durante a emissão de consoantes de pressão, podendo ou não ser audível. $\mathrm{O}$ enfraquecimento dos fonemas de pressão intraoral é caracterizado pela produção débil das consoantes plosivas e fricativas, devido à perda de ar para a cavidade nasal. Como efeito secundário da DVF na fala, encontra-se os distúrbios articulatórios compensatórios, que se caracterizam pela substituição do local de produção dos sons orais por outros no trato vocal, como a glote e as regiões da naso e orofaringe. Todas estas alterações combinadas acabam por prejudicar a inteligibilidade da fala ${ }^{(2,3,8,9)}$.

As técnicas para o tratamento cirúrgico da DVF podem ser divididas em cinco grandes grupos: aumento da parede posterior da faringe, mobilização dos músculos levantadores do palato (veloplastia intravelar), retroposição do palato (pushback), retalho faríngeo e esfincteroplastia ${ }^{(10,11)}$.

A técnica de retalho faríngeo de pedículo superior é uma das técnicas cirúrgicas mais utilizadas, consistindo no levantamento e um retalho miomucoso da parede posterior da faringe, inserido no palato mole, formando uma "ponte" de tecido, unindo o palato mole com a parede posterior da faringe. Lateralmente a este retalho, existem dois orifícios que permitirão a passagem de ar para a cavidade nasal na respiração e produção dos sons nasais. Durante a emissão dos sons orais, estes orifícios se fecham, como dois esfíncteres, para impedir o escape de ar nasal na fala. Essa técnica é geralmente utilizada para falhas no fechamento maiores do que $10 \mathrm{~mm}$ e na presença de movimento das paredes laterais da faringe, e tem se mostrado efetiva na correção ou diminuição da hipernasalidade da fala ${ }^{(12-14)}$.

A técnica de retalho faríngeo é indicada nos casos em que o padrão de movimento é do tipo circular ou no padrão sagital, em que se observa um deslocamento das paredes laterais da faringe ${ }^{(15,16)}$.

Vários trabalhos já investigaram os resultados cirúrgicos e funcionais da técnica de retalho faríngeo, mostrando que a melhora para a ressonância de fala equilibrada variou de $38 \%$ a $52 \%{ }^{(12,17)}$. Outro trabalho demonstrou que houve melhores resultados nos pacientes que realizaram fonoterapia após a cirurgia e nos que apresentavam hipernasalidade leve no préoperatório, porém, em alguns casos, ocorreu obstrução respiratória durante o sono após a cirurgia ${ }^{(12)}$. Outro estudo mostrou que o índice de insucesso foi de $25 \%$ dos casos, sendo que os autores atribuíram esse resultado a uma falha na execução do retalho em relação à largura, altura na parede posterior da faringe e simetria ${ }^{(18)}$. Outro autor observou que aqueles pacientes com padrão coronal de movimento velofaríngeo antes da cirurgia não apresentaram melhora tão significativa quanto aqueles com outros padrões de tentativa de fechamento ${ }^{(15)}$.

As técnicas que promovem a mobilização dos músculos levantadores do palato, ou veloplastia intravelar, são utilizadas quando os músculos levantadores do véu palatino estão inseridos na borda óssea posterior do palato duro, dificultando o fechamento velofaríngeo. O objetivo dessas técnicas cirúrgicas é promover a mudança na inserção dos músculos levantadores, deslocando-os para uma posição mais posterior no palato mole e com isso auxiliar a função palatina na fala. Elas geralmente são indicadas para falhas menores no fechamento velofaríngeo, ou seja, até $5 \mathrm{~mm}^{(19)}$.

Muitos estudos tiveram como objetivo avaliar os resultados da técnica de alongamento do palato. Todos concordam que esta técnica cirúrgica é mais eficiente na melhora e resolução dos sintomas de fala da DVF nos pacientes com pequenas falhas do fechamento ${ }^{(20-22)}$ e, após a correção cirúrgica pela veloplastia intravelar, há melhora significativa na ressonância de fala ${ }^{(19,23,24)}$.

Considerando a importância de se analisar os resultados funcionais da cirurgia secundária de correção da disfunção velofaríngea, o presente estudo teve como objetivo comparar os resultados da fala e da função velofaríngea após as técnicas de retalho faríngeo e veloplastia intravelar como procedimentos secundários para a correção da disfunção velofaríngea.

\section{MÉTODOS}

Esta pesquisa foi previamente aprovada pelo Comitê de Ética em Pesquisa com Seres Humanos do Hospital de Reabilitação de Anomalias Craniofaciais da Universidade de São Paulo - HRAC/USP, sob o protocolo número 175/2008.

\section{Casuística}

Foi realizado um estudo clínico retrospectivo no Setor de Fonoaudiologia do HRAC - USP, em que foram analisados 148 prontuários de pacientes com fissura labiopalatina operada e disfunção velofaríngea residual. Todos foram submetidos à correção cirúrgica da disfunção velofaríngea entre os anos de 1995 e 2007 na instituição, sendo que 77 foram submetidos à técnica de retalho faríngeo de pedículo superior (média de idade de 20,4 anos) e 71 submetidos às técnicas de veloplastia intravelar (média de idade de 16,2 anos).

Foram incluídos no estudo os prontuários que continham dados completos quanto à classificação da ressonância da fala, emissão de ar nasal e distúrbios articulatórios compensatórios antes e após as cirurgias, assim como a avaliação direta do mecanismo velofaríngeo, com nasofaringoscopia ou videofluoroscopia pré e pós-cirúrgica, independentemente do gênero, idade e tipo de fissura apresentada pelo indivíduo.

Foram excluídos os casos que apresentaram diagnóstico de síndrome genética que envolvesse alterações cognitivas ou de aprendizagem, e alterações anatômicas que pudessem afetar a avaliação da ressonância da fala, além dos casos com avaliações incompletas. 


\section{Avaliação perceptiva da fala}

A classificação da ressonância da fala e a identificação de presença ou ausência dos distúrbios articulatórios compensatórios foram realizadas por meio da avaliação perceptivo-auditiva de fonoaudiólogas com mais de dez anos de experiência no diagnóstico dos distúrbios da fala de indivíduos com fissura labiopalatina. Foi utilizada a fala dirigida, com a repetição de vocábulos e frases foneticamente balanceadas, e fala espontânea, em que o indivíduo foi estimulado a relatar fatos de seu cotidiano ou responder às perguntas feitas pela avaliadora.

O critério de classificação da ressonância antes da cirurgia foi: hipernasal leve não aceitável; hipernasal moderada; hipernasal grave. Na fase pós-cirúrgica, a classificação da ressonância foi: equilibrada; hipernasal leve aceitável; hipernasal leve não aceitável; hipernasal moderada; hipernasal grave; hiponasal aceitável; hiponasal não aceitável; mista. Esta classificação foi baseada na proposta de Henningsson et al. ${ }^{(25)}$, sendo que os graus de hipernasalidade leve aceitável e hiponasalidade aceitável são utilizados no Setor de Fonoaudiologia do Hospital de Reabilitação de Anomalias Craniofaciais da Universidade de São Paulo (HRAC-USP), pois representam uma melhora na ressonância da fala após a correção da DVF e são padrões de fala bem aceitos socialmente.

Os distúrbios articulatórios compensatórios foram considerados como presentes ou ausentes antes e após cada técnica cirúrgica.

A presença ou ausência de emissão de ar nasal foi avaliada com o auxílio de uma placa de inox posicionada logo abaixo das narinas do paciente, durante o sopro, emissão de fonemas fricativos isolados e vocábulos dissílabos com fonemas plosivos e fricativos. A presença de emissão nasal de ar foi caracterizada pelo embaçamento da superfície durante essas produções.

Foi considerada como melhora na ressonância da fala após a cirurgia, a redução de ao menos um nível na classificação.

\section{Avaliação objetiva do funcionamento velofaríngeo na fala}

Todos os prontuários apresentavam os resultados da avaliação nasofaringoscópica da velofaringe, antes e após a cirurgia, realizada por uma equipe composta por duas fonoaudiólogas e um cirurgião plástico com ampla experiência na área. Na avaliação pré-cirúrgica, foram considerados os seguintes padrões de tentativa de fechamento velofaríngeo: padrão circular (falha grande, média ou pequena); padrão circular com prega de Passavant (falha grande, média ou pequena); padrão transverso ou coronal (falha grande, média ou pequena); padrão sagital (falha grande, média ou pequena); fechamento marginal.

Para determinar o tamanho da falha no fechamento, utilizou-se como referência o diâmetro do orifício velofaríngeo durante o repouso e depois durante as emissões da fala. A presença de pouco movimento do véu palatino e das paredes da faringe com pequena variação em relação ao diâmetro da velofaringe no repouso foi considerada como uma falha grande, enquanto as falhas médias e pequenas corresponderam a maiores deslocamentos das estruturas da velofaringe e consequente redução do diâmetro do orifício velofaríngeo na fala.
$\mathrm{Na}$ avaliação nasofaringoscópica, realizada após a veloplastia intravelar, foram considerados os seguintes padrões: fechamento velofaríngeo total; falha grande no fechamento; falha média no fechamento; falha pequena no fechamento; fechamento marginal. Nos casos submetidos ao retalho faríngeo, foi considerado: fechamento total dos orifícios laterais ao retalho faríngeo; presença de falha no fechamento dos orifícios; ausência de movimento nos orifícios. Foi considerado como melhora pós-cirúrgica a redução de ao menos um grau no padrão de tentativa de fechamento.

A amostra de fala utilizada na nasofaringoscopia antes e após a cirurgia abrangeu as emissões de fonemas plosivos e fricativos surdos, vogais /a/, /i/ e /u/, vocábulos e frases com fonemas plosivos e fricativos. Para esse estudo, foram considerados apenas os dados da primeira avaliação perceptivoauditiva e nasofaringoscópica realizadas após a cirurgia, desde que tivessem sido efetuadas num período não inferior a seis meses após a cirurgia.

\section{Análise estatística}

Foi realizada análise descritiva dos instantes pré e póscirúrgico em relação à ressonância, distúrbio articulatório compensatório, emissão de ar nasal e padrão de movimento velofaríngeo. Também foi realizada a análise inferencial por meio do modelo de regressão logística ${ }^{(26)}$, para estudar a associação entre a melhora da ressonância, do distúrbio articulatório compensatório, da emissão de ar audível e do movimento velofaríngeo com as variáveis: técnica cirúrgica, tamanho da falha no fechamento velofaríngeo antes da cirurgia e faixa etária. Para avaliar a relação entre idade do indivíduo na época da cirurgia e o resultado da ressonância, optamos por separar aqueles com idades até 12 anos, que corresponde ao final da infância e início da adolescência, e indivíduos acima desta idade que hipoteticamente teriam menos chance de obter um bom resultado de fala.

\section{RESULTADOS}

A Tabela 1 mostra a distribuição da ressonância antes e após as técnicas de retalho faríngeo e veloplastia intravelar.

A análise estatística demonstrou, por meio do modelo de regressão logística, que os indivíduos com idade até 12 anos apresentaram em média 2,95 vezes a chance de melhorar a ressonância em relação ao grupo com idade superior a 12 anos, independente da técnica cirúrgica. Da mesma forma, pode-se afirmar que o grupo que se submete ao retalho faríngeo tem 3,99 vezes a chance de melhorar a ressonância em relação ao grupo que realiza a veloplastia (Tabela 2).

A Tabela 3 apresenta a distribuição da presença e ausência das articulações compensatórias antes e após as cirurgias de retalho faríngeo e veloplastia, não havendo diferença entre as duas técnicas. Entretanto, os indivíduos com idade até 12 anos apresentaram melhora significativa na produção das articulações compensatórias, independentemente da técnica cirúrgica. Observou-se nos dois grupos, sendo um caso de retalho faríngeo e um de veloplastia, a presença de articulação compensatória somente após as cirurgias. 
Tabela 1. Distribuição conjunta da ressonância pré e pós, de acordo com o tipo de cirurgia

\begin{tabular}{|c|c|c|c|c|c|c|c|c|c|c|c|c|c|c|c|c|c|}
\hline \multirow{2}{*}{ Cirurgia } & \multirow{2}{*}{$\begin{array}{c}\text { Ressonância } \\
\text { pré }\end{array}$} & \multirow[b]{2}{*}{$\mathrm{EQ}$} & \multirow[b]{2}{*}{$(\%)$} & \multirow[b]{2}{*}{ HLA } & \multirow[b]{2}{*}{$(\%)$} & \multirow[b]{2}{*}{ HLNA } & \multicolumn{4}{|c|}{ Ressonância pós } & \multirow[b]{2}{*}{$(\%)$} & \multirow[b]{2}{*}{$\mathrm{HO}$} & \multirow[b]{2}{*}{$(\%)$} & \multirow[b]{2}{*}{$\mathrm{M}$} & \multirow[b]{2}{*}{$(\%)$} & \multirow[b]{2}{*}{ Total } & \multirow[b]{2}{*}{$(\%)$} \\
\hline & & & & & & & $(\%)$ & $\mathrm{HM}$ & $(\%)$ & $H G$ & & & & & & & \\
\hline \multirow{4}{*}{ Retalho faríngeo } & HLNA & 9 & (12) & 2 & (3) & 1 & (1) & & & & & & & & & 12 & $(16)$ \\
\hline & $\mathrm{HM}$ & 8 & (10) & 8 & (10) & 17 & (22) & 12 & $(16)$ & & & 2 & (3) & 3 & $(4)$ & 50 & (65) \\
\hline & $H G$ & 2 & (3) & 1 & (1) & 5 & (6) & 5 & (6) & & & 2 & (3) & & & 15 & (19) \\
\hline & Total & 19 & $(25)$ & 11 & $(14)$ & 23 & $(30)$ & 17 & $(22)$ & & & 4 & $(5)$ & 3 & $(4)$ & 77 & $(100)$ \\
\hline \multirow{5}{*}{ Veloplastia } & HLA & 2 & (3) & & & & & & & & & & & & & 2 & (3) \\
\hline & HLNA & 15 & $(21)$ & 8 & (11) & 6 & (8) & & & & & & & 1 & (1) & 30 & $(42)$ \\
\hline & $\mathrm{HM}$ & 4 & (6) & 8 & (11) & 6 & (8) & 16 & (23) & & & & & & & 34 & (48) \\
\hline & $H G$ & & & & & 1 & (1) & 3 & (4) & 1 & (1) & & & & & 5 & (7) \\
\hline & Total & 21 & (30) & 16 & (23) & 13 & (18) & 19 & (27) & 1 & (1) & & & 1 & (1) & 71 & (100) \\
\hline
\end{tabular}

Legenda: $\mathrm{EQ}=$ equilibrada; HLA = hipernasal leve aceitável; HLNA = hipernasal leve não aceitável; HM = hipernasal moderada; HG = hipernasal grave; HO = hiponasal; $\mathrm{M}=$ mista

Tabela 2. Modelo de regressão logística - melhora da ressonância

\begin{tabular}{lccccc}
\hline Coeficientes & Estimativa & Erro-padrão & Valor de $\mathrm{p}$ & $\mathrm{RC}$ & $\begin{array}{c}\text { Intervalo de confiança (95\%) } \\
\text { Limite inferior }\end{array}$ \\
\hline Limite superior
\end{tabular}

Legenda: $\mathrm{RC}$ = razão de chances

Tabela 3. Distribuição das articulações compensatórias antes e após as cirurgias de retalho faríngeo e veloplastia

\begin{tabular}{|c|c|c|c|c|c|c|c|}
\hline \multirow{2}{*}{ Cirurgia } & \multirow{2}{*}{ DAC pré } & \multicolumn{4}{|c|}{ DAC pós } & \multirow{2}{*}{ Total } & \multirow{2}{*}{$(\%)$} \\
\hline & & Ausente & $(\%)$ & Presente & $(\%)$ & & \\
\hline \multirow{3}{*}{ Retalho faríngeo } & Ausente & 24 & (31) & 1 & $(1)$ & 25 & (32) \\
\hline & Presente & 6 & (8) & 47 & $(61)$ & 53 & $(69)$ \\
\hline & Total & 29 & (38) & 48 & $(62)$ & 78 & $(100)$ \\
\hline \multirow{3}{*}{ Veloplastia } & Ausente & 31 & $(44)$ & 1 & $(1)$ & 32 & $(46)$ \\
\hline & Presente & 2 & (3) & 36 & (51) & 38 & $(54)$ \\
\hline & Total & 33 & $(47)$ & 37 & (53) & 70 & (100) \\
\hline
\end{tabular}

Legenda: $\mathrm{DAC}=$ distúrbio articulatório compensatório

Em relação à emissão de ar nasal, nos instantes pré e póscirúrgico, não houve diferença entre as duas técnicas cirúrgicas (Tabela 4).

A técnica de retalho faríngeo proporcionou melhora significativa no padrão velofaríngeo, com 15,51 vezes a chance de melhora em relação ao grupo que realizou a veloplastia intravelar (Tabela 5).

Não houve diferença entre o tamanho da falha no fechamento antes da cirurgia e a melhora do padrão velofaríngeo observado após as cirurgias.

Tabela 4. Distribuição da emissão de ar nasal antes e após as técnicas cirúrgicas

\begin{tabular}{|c|c|c|c|c|c|c|c|}
\hline \multirow{2}{*}{ Cirurgia } & \multirow{2}{*}{ EAN pré } & \multicolumn{4}{|c|}{ EAN pós } & \multirow{2}{*}{ Total } & \multirow{2}{*}{$(\%)$} \\
\hline & & Ausente & (\%) & Presente & $(\%)$ & & \\
\hline \multirow{3}{*}{ Retalho faríngeo } & Ausente & 1 & (1) & 0 & (0) & 1 & (1) \\
\hline & Presente & 17 & (22) & 58 & (76) & 75 & (99) \\
\hline & Total & 18 & $(24)$ & 58 & $(76)$ & 76 & $(100)$ \\
\hline \multirow{3}{*}{ Veloplastia } & Ausente & 1 & (1) & 0 & (0) & 1 & (1) \\
\hline & Presente & 18 & $(26)$ & 51 & (73) & 69 & (99) \\
\hline & Total & 19 & (27) & 51 & (73) & 70 & (100) \\
\hline
\end{tabular}


Tabela 5. Distribuição do padrão velofaríngeo pré e pós de acordo com o tipo de cirurgia

\begin{tabular}{|c|c|c|c|c|c|c|c|c|c|c|c|c|c|c|c|c|c|}
\hline \multirow{2}{*}{ Grupo } & \multirow{2}{*}{$\begin{array}{l}\text { PVF } \\
\text { pré }\end{array}$} & \multicolumn{14}{|c|}{ PVF pós } & \multirow{2}{*}{ Total } & \multirow{2}{*}{$(\%)$} \\
\hline & & FMG & $(\%)$ & FT & $(\%)$ & $\mathrm{FF}$ & $(\%)$ & AF & $(\%)$ & $\mathrm{FP}$ & $(\%)$ & $\mathrm{FM}$ & $(\%)$ & $F G$ & $(\%)$ & & \\
\hline \multirow{12}{*}{$\begin{array}{l}\text { Retalho } \\
\text { faríngeo }\end{array}$} & FCP & & & 3 & (4) & & & & & & & & & & & 3 & (4) \\
\hline & FCM & & & 4 & (5) & 1 & (1) & & & & & & & & & 5 & (6) \\
\hline & FCG & 1 & (1) & 8 & (10) & 15 & (19) & 2 & (3) & & & & & & & 26 & (33) \\
\hline & FCPP & & & 1 & (1) & & & & & & & & & & & 1 & (1) \\
\hline & FCMP & & & 5 & (6) & 4 & (5) & & & & & & & & & 9 & (12) \\
\hline & FCGP & & & 11 & (14) & 9 & (12) & 1 & (1) & & & & & & & 21 & (27) \\
\hline & FTP & & & 2 & (3) & & & & & & & & & & & 2 & (3) \\
\hline & FTM & & & 3 & (4) & 1 & (1) & & & & & & & & & 4 & (5) \\
\hline & FTG & & & & & 1 & (1) & & & & & & & & & 1 & (1) \\
\hline & FSP & & & 1 & (1) & & & & & & & & & & & 1 & (1) \\
\hline & FMG & & & 4 & (5) & 1 & (1) & & & & & & & & & 5 & (6) \\
\hline & Total & 1 & (1) & 42 & (54) & 32 & (41) & 3 & (4) & & & & & & & 78 & (100) \\
\hline \multirow{10}{*}{ Velo-plastia } & FCP & & & 4 & (6) & & & & & 3 & (4) & 1 & (1) & 1 & (1) & 9 & (13) \\
\hline & FCM & & & 1 & (1) & & & & & 2 & (3) & 1 & (1) & & & 4 & (6) \\
\hline & FCG & 1 & (1) & 5 & (7) & & & & & 2 & (3) & 5 & (7) & 9 & (13) & 22 & (31) \\
\hline & FCPP & & & 2 & (3) & & & & & 2 & (3) & & & 1 & (1) & 5 & (7) \\
\hline & FCMP & & & 3 & (4) & & & & & & & 1 & (1) & & & 4 & (6) \\
\hline & FCGP & 1 & (1) & 2 & (3) & & & & & & & & & 4 & (6) & 7 & (10) \\
\hline & FTP & 1 & (1) & 1 & (1) & & & & & & & & & & & 2 & (3) \\
\hline & FTG & & & 1 & (1) & & & & & 2 & (3) & & & & & 3 & (4) \\
\hline & FMG & 3 & (4) & 9 & (13) & & & & & 2 & (3) & & & & & 14 & (20) \\
\hline & Total & 6 & (9) & 28 & $(40)$ & & & & & 13 & (19) & 8 & (11) & 15 & (21) & 70 & (100) \\
\hline
\end{tabular}

Legenda: $\mathrm{PVF}$ = padrão velofaríngeo; $\mathrm{FCP}$ = falha circular pequena; $\mathrm{FCM}$ = falha circular média; $F C G$ = falha circular grande; $F C P P=$ falha circular pequena com prega de Passavant; FCMP = falha circular média com Passavant; FCGP = falha circular grande com Passavant; FTP = falha transversa pequena; FTM = falha transversa média; $F T G$ = falha transversa grande; $F S P=$ falha sagital pequena; $F M G$ = falha marginal; $F T$ = fechamento total; $F F=$ falha no fechamento; $A F=$ ausência de fechamento; $F P$ = falha pequena; $F M=$ falha média; $F G$ falha grande

\section{DISCUSSÃO}

Este estudo se propôs a responder a algumas questões que surgem na prática clínica em relação ao tratamento cirúrgico da disfunção velofaríngea. As várias técnicas cirúrgicas utilizadas para esse fim têm sido descritas na literatura há alguns anos, assim como os parâmetros para a aplicação de cada uma delas de acordo com o grau da disfunção velofaríngea ${ }^{(10,11,27)}$.

A questão levantada no presente estudo refere-se aos resultados observados na fala após a técnica de retalho faríngeo de pedículo superior e as técnicas de veloplastia intravelar em nossa população, e à verificação de se uma técnica mostrouse mais efetiva que a outra na correção ou melhora da função velofaríngea na fala. Os resultados revelaram que os pacientes submetidos ao retalho faríngeo apresentaram $83 \%$ de melhora na ressonância, enquanto que aqueles submetidos à veloplastia intravelar apresentaram 68\% de melhora. Isto não significa necessariamente a normalização da ressonância, mas uma diminuição no grau de hipernasalidade. Dos $83 \%$ que melhoraram após o retalho faríngeo, $25 \%$ alcançaram a normalidade e 14\% permaneceram com uma hipernasalidade leve aceitável, enquanto que, entre os casos com veloplastia intravelar, $30 \%$ revelaram ressonância equilibrada e $23 \%$ permaneceram com hipernasalidade leve aceitável; portanto houve uma melhora satisfatória para ambas as técnicas.

Entretanto, a análise estatística comprovou que a técnica de retalho faríngeo proporciona uma chance de melhora de 3,99 vezes em relação à técnica de veloplastia intravelar, concordando com outro trabalho ${ }^{(28)}$. Este resultado difere de outro estudo, que não encontrou diferença na ressonância entre as duas técnicas cirúrgicas ${ }^{(23)}$. É fato que a técnica de retalho faríngeo ocasiona maior obstrução na nasofaringe ${ }^{(29,30)}$, portanto, as chances de diminuição da hipernasalidade são maiores quando comparada à veloplastia intravelar, que é uma técnica mais fisiológica e menos obstrutiva. Com a maior oclusão do orifício velofaríngeo provocada pelo retalho faríngeo, a probabilidade de uma hipercorreção e consequente hiponasalidade são maiores. Nos resultados do presente estudo foi verificado que apenas $6 \%$ dos casos submetidos a essa técnica apresentaram hiponasalidade na fala, assim como em outro estudo que encontrou o mesmo resultado ${ }^{(24)}$.

Com relação à idade, o presente estudo revelou maiores chances de melhora na ressonância em crianças até 12 anos, fato também demonstrado por outro estudo ${ }^{(19)}$. Isto comprova 
a importância da correção da DVF na infância, período de crescimento e maior plasticidade do sistema sensório-motor, favorecendo o desenvolvimento de uma função velofaríngea normal na fala e evitando problemas psicossociais. O presente estudo revelou que não houve mudança significativa na presença de articulações compensatórias antes e após as duas técnicas cirúrgicas, comprovando que a cirurgia para a DVF não tem a função de eliminar esses distúrbios, mas promover condições anatomofuncionais que favoreçam sua eliminação com a fonoterapia. Os dois casos em que houve a presença de articulação compensatória somente após as cirurgias podem ser explicados pela não identificação do distúrbio antes da cirurgia pela fonoaudióloga, ou pela hipernasalidade e fraca pressão, que teriam dificultado a identificação da articulação compensatória, que ficou evidenciada após a melhora da ressonância.

Analisando a presença de emissão de ar nasal detectável na placa de inox, foram encontrados neste estudo valores elevados após as duas técnicas cirúrgicas, pois esse sinal pode ser encontrado em pequenas falhas no fechamento velofaríngeo e na presença de ressonância de fala aceitável. Isto explicaria a presença elevada de emissão de ar nasal com bons resultados na ressonância da fala.

O padrão de fechamento velofaríngeo é um fator determinante na indicação da técnica cirúrgica para correção da DVF, uma vez que o tamanho da falha e o grau de deslocamento das estruturas da velofaringe indicarão qual o procedimento mais adequado.

A literatura aponta que a técnica de retalho faríngeo pode ser indicada em falhas grandes no fechamento e padrões de tentativa de fechamento do tipo circular ou sagital, pois em ambos existe movimento das paredes laterais da faringe, condição importante para o fechamento dos orifícios laterais ao retalho faríngeo ${ }^{(10,11,15,16)}$. No presente estudo, foi constatado que os indivíduos submetidos à técnica de retalho faríngeo apresentaram melhora significativa no padrão velofaríngeo, com consequente diminuição da hipernasalidade. Assim como na literatura, a população retratada neste estudo apresentou 90\% de pacientes com falhas médias a grandes no fechamento antes da técnica de retalho faríngeo, enquanto $57 \%$ do grupo com veloplastia intravelar apresentou os mesmos tamanhos de falha. Entre os pacientes que apresentaram falhas médias e grandes antes da veloplastia, foi verificado que apenas $16 \%$ apresentaram fechamento velofaríngeo total, enquanto que os $90 \%$ submetidos ao retalho faríngeo apresentaram fechamento total em $39 \%$ dos casos.

Pode-se inferir que os resultados poderiam ter sido melhores no grupo com veloplastia, caso não fossem incluídos pacientes com falhas maiores no fechamento. Alguns estudos mostraram que as técnicas de veloplastia intravelar apresentam melhores resultados em pacientes com falhas menores no fechamento ${ }^{(20,22)}$.

Dessa forma, baseados nos dados obtidos, acredita-se que a definição da conduta cirúrgica para os casos com falhas médias e grandes no fechamento deve considerar o procedimento cirúrgico que oclua mais efetivamente a velofaringe, como o retalho faríngeo, aumentando as chances de correção da DVF.

\section{CONCLUSÃO}

Os resultados obtidos no presente estudo permitem concluir que a técnica de retalho faríngeo promoveu uma melhora significativa na ressonância da fala e na função velofaríngea quando comparada à técnica de veloplastia intravelar. As duas técnicas cirúrgicas não diferiram em relação à emissão de ar nasal e articulações compensatórias.

\begin{abstract}
Purpose: To compare speech and velopharyngeal function after pharyngeal flap and intravelar veloplasty techniques for the correction of residual velopharyngeal dysfunction. Methods: It was carried out a retrospective study analyzing 148 cases of cleft lip and palate operated and submitted to velopharyngeal dysfunction surgical correction, 77 with pharyngeal flap (mean age: 20.4 years) and 71 with intravelar veloplasty (mean age: 16.2 years). Speech resonance, presence of compensatory articulations, nasal air emission, and velopharyngeal gap size were assessed before and after the use of both techniques. Results: Sixty four (83\%) of the 77 cases submitted to pharyngeal flap presented resonance improvement, while $48(68 \%)$ of the 71 cases with intravelar veloplasty improved, with significant difference between the groups. Six (8\%) subjects with pharyngeal flap, and two (3\%) with intravelar veloplasty showed improvement in compensatory articulations, while nasal air emission improved in 17 (22\%) cases with pharyngeal flap, and $18(26 \%)$ with intravelar veloplasty. No significant differences were found between the groups regarding compensatory articulations and nasal air emission. The velopharyngeal gap size reduced in 75 (96\%) cases with pharyngeal flap, and 46 (66\%) with intravelar veloplasty, with a significant difference between the groups. Conclusion: The pharyngeal flap technique was more effective in improving resonance and velopharyngeal closure, when compared to intravelar veloplasty.
\end{abstract}

Keywords: Cleft lip/surgery; Cleft palate/surgery; Velopharyngeal insufficiency/therapy; Speech disorders; Palate/surgery 


\section{REFERÊNCIAS}

1. Bzoch KR. Introduction to the study of communicative disorders in cleft palate and related craniofacial anomalies. In: Bzoch KR, editor. Communicative disorders related to cleft lip and palate. 5th ed. Austin: Pro-Ed; c2004. p. 3-65.

2. Kummer AW. Velopharyngeal dysfunction and resonance disorders. In: Kummer AW. Cleft palate and craniofacial anomalies: the effects on speech and resonance. San Diego: Singular; c2001. p . 145-76.

3. Golding-Kushner KJ. Therapy techniques for cleft palate speech and related disorders. San Diego: Singular; c2001. p. 13-34.

4. Skolnick ML, McCall GN, Barnes M. The sphincteric mechanism of velopharyngeal closure. Cleft Palate J. 1973;10:286-305.

5. Hudson DA, Grobbelaar AO, Fernandes DB, Lentin R. Treatment of velopharyngeal incompetence by the Furlow Z-plasty. Ann Plast Surg. 1995;34(1):23-6.

6. Dreyer TM, Trier WC. A comparison of palatoplasty techniques. Cleft Palate J. 1984;21(4):251-3.

7. Aaronson SM, Fox DR, Cronin TD. The Cronin push-back palate repair with nasal mucosal flaps: a speech evaluation. Plast Reconstr Surg. 1985;75(6):805-9.

8. Trost JE. Articulatory additions to the classical description of the speech of persons with cleft palate. Cleft Palate J. 1981;18(3):193-203.

9. Trost-Cardamone JE. Effects of velopharyngeal incompetence on speech. Commun Disord Q. 1986;10(1):31-49.

10. Bardach J. Secondary surgery for velopharyngeal insufficiency. In: Shprintzen RJ, Bardach J, editors. Cleft palate speech management: a multidisciplinary approach. St. Louis: Mosby; c1995. p. 277-94.

11. Riski JE. Secondary surgical procedures to correct postoperative velopharyngeal incompetencies found after primary palatoplasties. In: Bzoch KR, editor. Communicative disorders related to cleft lip and palate. 5th ed. Austin: Pro-Ed; c2004. p.193-236.

12. Canady JW, Cable BB, Karnell MP, Karnell LH. Pharyngeal flap surgery: protocols, complications, and outcomes at the University of Iowa. Otolaryngol Head Neck Surg. 2003;129(4):321-6.

13. Cable B, Canady JW, Karnell MP, Karnell LH, Malick DN. Pharyngeal flap surgery: long-term outcomes at the University of Iowa. Plast Reconstr Surg. 2004;113(2):475-8.

14. D'Antonio LL. Evaluation and management of velopharyngeal dysfunction: a speech pathologist's viewpoint. Probl Plast Reconstr Surg. 1992;2:86-111.

15. Armour A, Fischbach S, Klaiman P, Fisher DM. Does velopharyngeal closure pattern affect the success of pharyngeal flap pharyngoplasty? Plast Reconstr Surg. 2005;115(1):45-52; discussion 53.

16. Liedman-Boshko J, Lohmander A, Persson C, Lith A, Elander A. Perceptual analysis of speech and the activity in the lateral pharyngeal walls before and after velopharyngeal flap surgery. Scand J Plast Reconstr Surg Hand Surg. 2005;39(1):22-32.
17. Fukushiro AP. Análise perceptiva, nasométrica e aerodinâmica da fala de indivíduos submetidos à cirurgia de retalho faríngeo para a correção da insuficiência velofaríngea [tese]. Bauru: Hospital de Anomalias Craniofaciais da Universidade de São Paulo; 2007.

18. Argamaso RV, Shprintzen RJ, Strauch B, Lewin ML, Daniller AI, Ship AG, Croft CB. The role of lateral pharyngeal wall movement in pharyngeal flap surgery. Plast Reconstr Surg. 1980;66(2):214-9.

19. Furlow LT Jr. Cleft palate repair by double opposing Z-plasty. Plast Reconstr Surg. 1986;78(6):724-38.

20. Perkins JA, Lewis CW, Gruss JS, Eblen LE, Sie KC. Furlow palatoplasty for management of velopharyngeal insufficiency: a prospective study of 148 consecutive patients. Plast Reconstr Surg. 2005;116(1):72-80; discussion 81-4.

21. Von Langenbeck B. Uranoplastik mittelst Ablosung des mucosperiostalen Gaumenuberzuges. Arch Klin Chir. 1861;2:205.

22. Carvalho ELL. Resultado de fala em pacientes submetidos à palatoplastia secundária associada à veloplastia intravelar [tese]. Bauru: Hospital de Reabilitação de Anomalias Craniofaciais da Universidade de São Paulo; 2006.

23. Noorchashm N, Dudas JR, Ford M, Gastman B, Deleyiannis FW, Vecchione L, et al. Conversion Furlow palatoplasty: salvage of speech after straight-line palatoplasty and "incomplete intravelar veloplasty". Ann Plast Surg. 2006;56(5):505-10.

24. Dailey SA, Karnell MP, Karnell LH, Canady JW. Comparison of resonance outcomes after pharyngeal flap and furlow double-opposing Z-plast for surgical management of velopharyngeal incompetence. Cleft Palate Craniofac J. 2006;43(1):38-43.

25. Henningsson G, Kuehn DP, Sell D, Sweeney T, Trost-Cardamone JE, Whitehill TL; Speech Parameters Group. Universal parameters for reporting speech outcomes in individuals with cleft palate. Cleft Palate Craniofac J. 2008;45(1):1-17.

26. Hosmer DW, Lemeshow S. Applied logistic regression. New York: Wiley; c1989. p. 307.

27. Khosla RK, Mabry K, Castiglione CL. Clinical outcomes of the Furlow Z-plasty for primary cleft palate repair. Cleft Palate Craniofac J. 2008;45(5):501-10.

28. Lesavoy MA, Borud LJ, Thorson T, Riegelhuth ME, Berkowitz CD. Upper airway obstruction after pharyngeal flap surgery. Ann Plast Surg. 1996;36(1):26-30; discussion 31-2.

29. Peña M, Choi S, Boyajian M, Zalzal G. Perioperative airway complications following pharyngeal flap palatoplasty. Ann Otol Rhinol Layngol. 2000;109(9):808-11.

30. Wells MD, Vu TA, Luce EA. Incidence and sequelae of nocturnal respiratory obstruction following posterior pharyngeal flap operation. Ann Plast Surg. 1999;43(3):252-7. 